\title{
sciendo
}

Int. J. of Applied Mechanics and Engineering, 2019, vol.24, No.3, pp.591-602

DOI: 10.2478/ijame-2019-0037

\section{TRAJECTORY PLANNING OF THE HUMANOID MANIPULATOR}

\author{
C. KACPRZAK* and G. PAJĄK \\ University of Zielona Góra \\ Institute of Mechanical Engineering and Machine Operation \\ Licealna 9, 65-417 Zielona Góra, POLAND \\ E-mails: c.cebula@o2.pl; g.pajak@iizp.uz.zgora.pl
}

\begin{abstract}
The paper presents a method of planning a collision-free trajectory for a humanoid manipulator mounted on a rail system. The task of the robot is to move its end-effectors from the current position to the given final location in the workspace. The method is based on a redundancy resolution at the velocity level. In addition to this primary task, secondary objectives are also taken into account. The motion of the robot is planned in order to maximize a manipulability measure in purpose of avoiding manipulator singularities. State inequality constraints resulting from collision avoidance conditions are also considered. A computer example involving a humanoid manipulator operating in a three dimensional task space is also presented.
\end{abstract}

Key words: humanoid manipulator, redundancy resolution, collision avoidance, manipulability measure.

\section{Introduction}

Nowadays serial manipulators are widely used in industry, they are employed to carry out various tasks such as pick and place operations, assembly, welding, spray painting and many others. However, there are also a lot of tasks that cannot be accomplished with a single manipulator arm. In many cases it is necessary to perform complex manipulation tasks in which two coordinated arms are needed. Amongst others, they include for example screw assembly or peg-in-hole tasks. It results in growing interest in the application of humanoid manipulators (Harada et al. [1]). The methods used to motion planning of dual arm robots are based on solutions for serial manipulators. They are based on kinematic redundancy resolution using the Jacobian pseudo-inverse (Caccavale et al. [2], Caccavale et al. [3]), augmented Jacobian (Vahrenkamp et al. [4]) and relative Jacobian (Jamisola et al. [5]). In some approaches dynamics of the system is taken into account and dynamic controllers are proposed (Caccavale et al. [3], Dietrich et al. [6]).

In this paper, the task of the humanoid manipulator is to pick parts from the storage location (which then have to be delivered to the assembly line). To increase its workspace it has been mounted on a rail system - so in this case the robot can be used to retrieve parts in the kitting system. Kitting is a method based on kit preparation and delivery of parts to feeding assembly lines. An economic comparison of different kinds of kitting systems has been carried out in (Caputo et al. [7]). The authors showed that automated (or robotic) retrieval solutions are cost effective as compared to manual ones. Boudella et al. [8] have estimated performance of the kitting system with the robot arm mounted on a rail system which has moved along the picking aisle and then has picked parts. To design such a system the knowledge of robotics and observations made on current kitting areas, discussions with experts and company workers are also needed (Patalas-Maliszewska [9]).

\section{Model of the system}

In the paper a humanoid manipulator mounted on a rail system moving along the picking aisle is considered. The humanoid manipulator is inspired by the upper body of the Justin robot developed by the German Aerospace Center (DLR) and it is composed of a three DOFs torso and two four DOFs manipulators (arms of the original Justin robot consist of manipulators with seven DOFs). Additionally, to increase the

\footnotetext{
* To whom correspondence should be addressed
} 
operating range of the robot, it is mounted on a rail system. A kinematic scheme of the whole robotic system is shown in Fig.1.

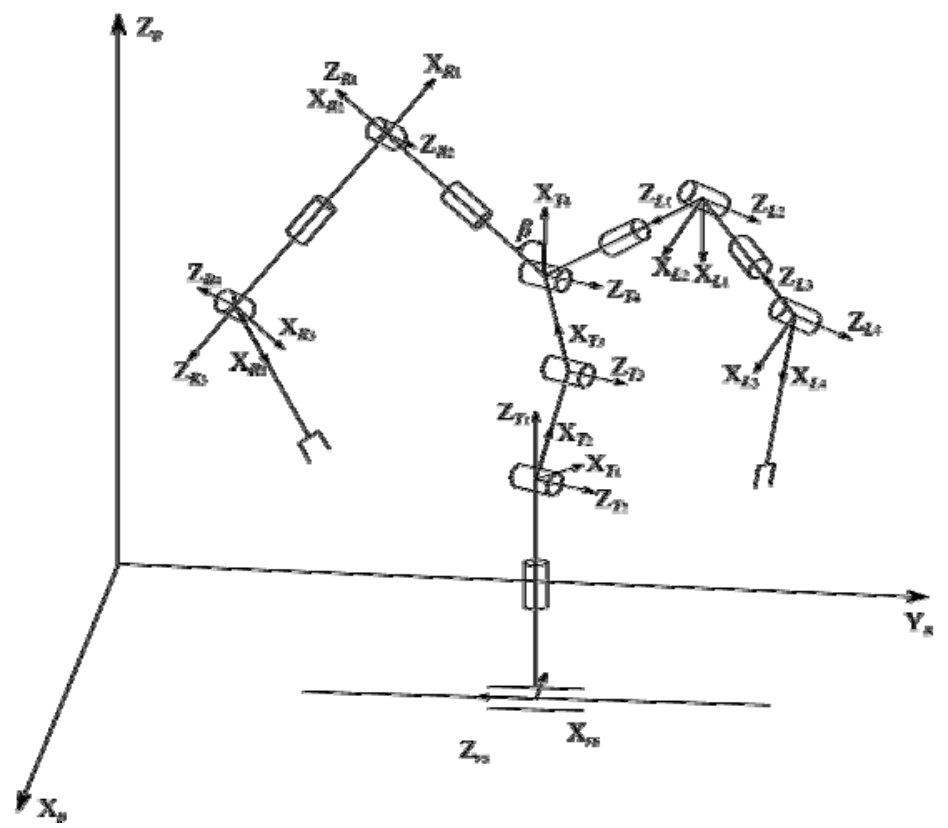

Fig.1. Kinematic scheme of the robot.

As it can be seen in the figure the kinematic model of the robot consists of one prismatic and twelve revolute joints. The first prismatic joint is used to allow the robot to move along the rail, the next revolute joints are associated with rotational movements performed by the torso and both arms of the humanoid. The kinematic structure of the robotic system has thirteen joints but it has only twelve DOFs:

- 1 DOF is connected to the movement along the rail,

- 3 DOFs are introduced by the torso but as it can be seen in Fig.1, the torso is modeled using four revolute joints, the last joint located at the end of the torso is passive and it ensures that the chest is always kept orthogonal to the ground (a configuration parameter connected to this joint is used to compensate rotations introduced by two previous joints),

- two arms, each with 4 DOFs.

It is assumed that the humanoid robot consists of series of rigid bodies connected by rigid joints. In this case, the location of each link with respect to a previous link, can be easily described by the location (position and orientation) of the local coordinate frame, attached rigidly to the chosen link, relative to the local coordinate frame attached to the previous link. To increase the readability of the presentation, the $\mathrm{Y}$ axes in Fig. 1 have been omitted, but they are chosen to complete right-handed coordinate frames.

In order to describe the position of the two end-effectors (left and right 'hand') of the robot in the base frame $O_{B} X_{B} Y_{B} Z_{B}$, the homogeneous transformation matrix approach and the modified DenavitHartenberg (DH) notation introduced by Craig [10] is used (axis $Z_{i}$ of a local coordinate frame is located along the axis of joint $i$ ). As it can be seen in Fig.1, the frame $O_{r s} X_{r s} Y_{r s} Z_{r s}$ is attached to the base of the torso, position of the origin of this frame describes the robot movement along the rail. Frames $O_{T_{1}} X_{T_{1}} Y_{T_{1}} Z_{T_{I}}$ $O_{T_{4}} X_{T_{4}} Y_{T_{4}} Z_{T_{4}}$ are assigned to the joints of the torso, $O_{L_{I}} X_{L_{1}} Y_{L_{I}} Z_{L_{I}}-O_{L_{4}} X_{L_{4}} Y_{L_{4}} Z_{L_{4}}$ and $O_{R_{I}} X_{R_{I}} Y_{R_{I}} Z_{R_{1}}$ $O_{R_{4}} X_{R_{4}} Y_{R_{4}} Z_{R_{4}}$ to the joints of the left and the right arm of the robot, respectively. The DH notation is applicable to serial open chain manipulators so in the case of a humanoid robot, which has a tree kinematic structure, it can be used to describe each of two chains starting from the base and ending with the end-effectors of the robot or to describe kinematics of the torso and each arm separately. The DH parameters of the robot are 
specified in Tab.1. (parameters of the arms have been obtained taking into account that the base frames of the arms are located at the end of the torso and they are rotated by the angle of $\beta$ as shown in Fig.1).

Table 1. DH parameters for the humanoid manipulator.

\begin{tabular}{|l|l|l|l|l|}
\hline \multicolumn{4}{|l|}{ torso } \\
\hline$i$ & $\alpha_{i-1}$ & $a_{i-1}$ & $d_{i}$ & $\theta_{i}$ \\
\hline 1 & $\frac{\pi}{2}$ & 0 & $l_{T_{1}}$ & $q_{2}$ \\
\hline 2 & $\frac{\pi}{2}$ & 0 & 0 & $q_{3}$ \\
\hline 3 & 0 & $l_{T_{2}}$ & 0 & $q_{4}$ \\
\hline 4 & 0 & $l_{T_{3}}$ & 0 & $q^{*}$ \\
\hline
\end{tabular}

\begin{tabular}{|l|l|l|l|l|}
\hline \multicolumn{4}{|l|}{ left arm } \\
\hline$i$ & $\alpha_{i-1}$ & $a_{i-1}$ & $d_{i}$ & $\theta_{i}$ \\
\hline 1 & $\pi$ & 0 & $-l_{L_{1}}$ & $q_{5}$ \\
\hline 2 & $\frac{\pi}{2}$ & 0 & 0 & $q_{6}$ \\
\hline 3 & $-\frac{\pi}{2}$ & 0 & $-l_{L_{2}}$ & $q_{7}$ \\
\hline 4 & $\frac{\pi}{2}$ & 0 & 0 & $q_{8}$ \\
\hline
\end{tabular}

\begin{tabular}{|l|l|l|l|l|}
\hline \multicolumn{6}{|l|}{ right arm } \\
\hline$i$ & $\alpha_{i-1}$ & $a_{i-1}$ & $d_{i}$ & $\theta_{i}$ \\
\hline 1 & 0 & 0 & $l_{R_{1}}$ & $q_{9}$ \\
\hline 2 & $-\frac{\pi}{2}$ & 0 & 0 & $q_{10}$ \\
\hline 3 & $\frac{\pi}{2}$ & 0 & $l_{R_{2}}$ & $q_{11}$ \\
\hline 4 & $-\frac{\pi}{2}$ & 0 & 0 & $q_{12}$ \\
\hline
\end{tabular}

where: $l_{T_{1}}, l_{T_{2}}, l_{T_{3}}, l_{L_{1}}, l_{L_{2}}, l_{R_{1}}, l_{R_{2}}$ are lengths of humanoid links, $q_{2}, q_{3}, q_{4}$ are joint angles of the torso, $q_{5}, q_{6}, q_{7}, q_{8}$ are joint angles of the left arm and $q_{9}, q_{10}, q_{11}, q_{12}$ are joint angles of the right arm, $q^{*}$ is a joint angle of the last joint of the torso, it used to compensate rotations introduced by two previous joints, $q^{*}=\frac{\pi}{2}-q_{3}-q_{4}$.

Taking into account the kinematic scheme of the robot and DH parameters defined in Tab.1, the homogeneous transformation matrices describing the location of each link with respect to a previous link can be determined as

$$
\begin{aligned}
& { }_{r s}^{B} \boldsymbol{T}=\left[\begin{array}{rrrr}
1 & 0 & 0 & 0 \\
0 & 0 & 1 & q_{1} \\
0 & -1 & 0 & 0 \\
0 & 0 & 0 & 1
\end{array}\right], \quad{ }_{T}^{r s} \boldsymbol{T}=\left[\begin{array}{cccc}
c_{q_{2}} & -s_{q_{2}} & 0 & 0 \\
0 & 0 & -1 & -l_{T_{1}} \\
s_{q_{2}} & c_{q_{2}} & 0 & 0 \\
0 & 0 & 0 & 1
\end{array}\right], \quad T_{T_{2}} \boldsymbol{T}=\left[\begin{array}{cccc}
c_{q_{3}} & -s_{q_{3}} & 0 & 0 \\
0 & 0 & -1 & 0 \\
s_{q_{3}} & c_{q_{3}} & 0 & 0 \\
0 & 0 & 0 & 1
\end{array}\right], \quad{ }_{T_{3}}^{T_{2}} \boldsymbol{T}=\left[\begin{array}{cccc}
c_{q_{4}} & -s_{q_{4}} & 0 & l_{T_{2}} \\
s_{q_{4}} & c_{q_{4}} & 0 & 0 \\
0 & 0 & 1 & 0 \\
0 & 0 & 0 & 1
\end{array}\right], \\
& { }_{T_{4}}^{T_{3}} \boldsymbol{T}=\left[\begin{array}{cccc}
c_{q^{*}} & -s_{q^{*}} & 0 & l_{T_{3}} \\
s_{q^{*}} & c_{q^{*}} & 0 & 0 \\
0 & 0 & 1 & 0 \\
0 & 0 & 0 & 1
\end{array}\right], \quad{ }_{L_{0}}^{T_{4}} \boldsymbol{T}=\left[\begin{array}{cccc}
-s_{\beta} & 0 & c_{\beta} & 0 \\
0 & -1 & 0 & 0 \\
c_{\beta} & 0 & s_{\beta} & 0 \\
0 & 0 & 0 & 1
\end{array}\right], \quad{ }_{L}^{L_{0}} \boldsymbol{T}=\left[\begin{array}{cccc}
c_{q_{5}} & -s_{q_{5}} & 0 & 0 \\
-s_{q_{5}} & -c_{q_{5}} & 0 & 0 \\
0 & 0 & -1 & 0 \\
0 & 0 & 0 & 1
\end{array}\right],{ }_{L_{2}}^{L_{1}} \boldsymbol{T}=\left[\begin{array}{cccc}
c_{q_{6}} & -s_{q_{6}} & 0 & 0 \\
0 & 0 & -1 & 0 \\
s_{q_{6}} & c_{q_{6}} & 0 & 0 \\
0 & 0 & 0 & 1
\end{array}\right], \\
& { }_{L_{3}}^{L_{2}} \boldsymbol{T}=\left[\begin{array}{cccc}
c_{q_{7}} & -s_{q_{7}} & 0 & 0 \\
0 & 0 & 1 & -l_{L_{2}} \\
-s_{q_{7}} & -c_{q_{7}} & 0 & 0 \\
0 & 0 & 0 & 1
\end{array}\right],{ }_{L_{4}}^{L_{3}} \boldsymbol{T}=\left[\begin{array}{cccc}
c_{q_{8}} & -s_{q_{8}} & 0 & 0 \\
0 & 0 & -1 & 0 \\
s_{q_{8}} & c_{q_{8}} & 0 & 0 \\
0 & 0 & 0 & 1
\end{array}\right], \quad T_{4} \boldsymbol{T}=\left[\begin{array}{cccc}
-s_{\beta} & 0 & c_{\beta} & 0 \\
0 & 1 & 0 & 0 \\
-c_{\beta} & 0 & -s_{\beta} & 0 \\
0 & 0 & 0 & 1
\end{array}\right], \quad R_{R_{l}}^{R_{0}} \boldsymbol{T}=\left[\begin{array}{cccc}
c_{q_{9}} & -s_{q_{9}} & 0 & 0 \\
s_{q_{9}} & c_{q_{9}} & 0 & 0 \\
0 & 0 & 1 & l_{L_{1}} \\
0 & 0 & 0 & 1
\end{array}\right], \\
& { }_{R_{2}}^{R_{1}} \boldsymbol{T}=\left[\begin{array}{cccc}
c_{q_{10}} & -s_{q_{10}} & 0 & 0 \\
0 & 0 & 1 & 0 \\
-s_{q_{10}} & -c_{q_{10}} & 0 & 0 \\
0 & 0 & 0 & 1
\end{array}\right], \quad{ }_{R_{3}}^{R_{2}} \boldsymbol{T}=\left[\begin{array}{cccc}
c_{q_{11}} & -s_{q_{11}} & 0 & 0 \\
0 & 0 & -1 & -l_{R_{2}} \\
s_{q_{11}} & c_{q_{11}} & 0 & 0 \\
0 & 0 & 0 & 1
\end{array}\right],{ }_{R_{4}}^{R_{3}} \boldsymbol{T}=\left[\begin{array}{cccc}
c_{q_{12}} & -s_{q_{12}} & 0 & 0 \\
0 & 0 & 1 & 0 \\
-s_{q_{12}} & -c_{q_{12}} & 0 & 0 \\
0 & 0 & 0 & 1
\end{array}\right],
\end{aligned}
$$

where $c_{\text {angle }}=\cos ($ angle $), s_{\text {angle }}=\sin ($ angle $),{ }_{i}^{j} \boldsymbol{T}$ is the homogeneous transformation matrix describing the position and orientation of the $O_{i} X_{i} Y_{i} Z_{i}$ frame in the $O_{j} X_{j} Y_{j} Z_{j}$ frame. 
Positions of the end-effectors expressed in the coordinate frame of the last joint of the left and the right arm are given as

$$
{ }^{L_{4}} \boldsymbol{p}_{L}=\left[\begin{array}{c}
l_{L_{3}} \\
0 \\
0
\end{array}\right] \quad \text { and } \quad{ }^{R_{4}} \boldsymbol{p}_{R}=\left[\begin{array}{c}
l_{R_{3}} \\
0 \\
0
\end{array}\right],
$$

where $l_{L_{3}}, l_{R_{3}}$ are lengths of the end-effectors of the humanoid manipulator and ${ }^{i} \boldsymbol{p}$ is the position of a specified point expressed in the $O_{i} X_{i} Y_{i} Z_{i}$ frame.

Positions of the end-effectors expressed in the base frame can be found after composition of transformations as follows

$$
\begin{aligned}
& { }^{B} \boldsymbol{p}_{L}={ }_{r s}^{B} \boldsymbol{T}_{T_{1}}^{r_{S}} \boldsymbol{T}_{T_{2}}^{T_{1}} \boldsymbol{T}_{T 3}^{T_{2}} \boldsymbol{T}_{T_{4}}^{T_{3}} \boldsymbol{T}_{L_{0}}^{T_{4}} \boldsymbol{T}_{L_{1}}^{L_{0}} \boldsymbol{T}_{L_{2}}^{L_{1}} \boldsymbol{T}_{L_{3}}^{L_{2}} \boldsymbol{T}_{L_{4}}^{L_{3}} \boldsymbol{T}^{L_{4}} \boldsymbol{p}_{L}, \\
& { }^{B} \boldsymbol{p}_{R}={ }_{r S}^{B} \boldsymbol{T}_{T_{1}}^{r_{S}} \boldsymbol{T}_{T_{2}}^{T_{1}} \boldsymbol{T}_{T 3}^{T_{2}} \boldsymbol{T}_{T_{4}}^{T_{3}} \boldsymbol{T}_{R_{0}}^{T_{4}} \boldsymbol{T}_{R_{1}}^{R_{0}} \boldsymbol{T}_{R_{2}}^{R_{1}} \boldsymbol{T}_{R_{3}}^{R_{2}} \boldsymbol{T}_{R_{4}}^{R_{3}} \boldsymbol{T}^{R_{4}} \boldsymbol{p}_{R} .
\end{aligned}
$$

Introducing the vectors of generalized coordinates of the torso, the left and right arm and the whole system as

$$
\boldsymbol{q}_{T}=\left[\begin{array}{l}
q_{1} \\
q_{2} \\
q_{3} \\
q_{4}
\end{array}\right], \quad \boldsymbol{q}_{L}=\left[\begin{array}{l}
q_{5} \\
q_{6} \\
q_{7} \\
q_{8}
\end{array}\right], \quad \boldsymbol{q}_{R}=\left[\begin{array}{c}
q_{9} \\
q_{10} \\
q_{11} \\
q_{12}
\end{array}\right], \quad \boldsymbol{q}=\left[\begin{array}{c}
\boldsymbol{q}_{T} \\
\boldsymbol{q}_{L} \\
\boldsymbol{q}_{R}
\end{array}\right],
$$

and ignoring orientations of the end effectors, the kinematic model of the system can be described as

$$
\boldsymbol{x}=\boldsymbol{f}(\boldsymbol{q})=\left[\begin{array}{l}
\boldsymbol{f}_{L}(\boldsymbol{q}) \\
\boldsymbol{f}_{R}(\boldsymbol{q})
\end{array}\right],
$$

where: $\boldsymbol{x} \in \mathfrak{R}^{6}$ is the vector with positions of the humanoids end-effectors expressed in the base frame, $\boldsymbol{f}(\boldsymbol{q})$ is the vector function determining the humanoid manipulator kinematic model, $\boldsymbol{f}: \mathfrak{R}^{12} \rightarrow \mathfrak{R}^{6}$ and $\boldsymbol{f}_{L}(\boldsymbol{q}), \boldsymbol{f}_{R}(\boldsymbol{q})$ are the functions describing positions of the left and right end-effectors in the base frame.

The end-effectors linear velocities in the base frame are given by the differential kinematics equation

$$
\dot{\boldsymbol{x}}=\boldsymbol{J}(\boldsymbol{q}) \dot{\boldsymbol{q}},
$$

where $\dot{\boldsymbol{x}}$ is the vector containing linear velocities of the end-effectors, $\dot{\boldsymbol{q}}$ is the vector of the generalized velocities of the robot joints and $\boldsymbol{J}=\partial \boldsymbol{f}(\boldsymbol{q}) / \partial \boldsymbol{q}$ is the $(6 \times 12)$ dimensional Jacobian matrix of the humanoid manipulator

$$
\boldsymbol{J}(\boldsymbol{q})=\left[\begin{array}{ccc}
\boldsymbol{J}_{L, \text { torso }} & \boldsymbol{J}_{L, \text { larm }} & \boldsymbol{0} \\
\boldsymbol{J}_{R, \text { torso }} & \boldsymbol{0} & \boldsymbol{J}_{R, \text { rarm }}
\end{array}\right]
$$

where $\boldsymbol{J}_{L, \text { torso }}=\partial \boldsymbol{f}_{L}(\boldsymbol{q}) / \partial \boldsymbol{q}_{T}, \boldsymbol{J}_{L, \text { larm }}=\partial \boldsymbol{f}_{L}(\boldsymbol{q}) / \partial \boldsymbol{q}_{L}, \boldsymbol{J}_{R, \text { torso }}=\partial \boldsymbol{f}_{R}(\boldsymbol{q}) / \partial \boldsymbol{q}_{T}, \boldsymbol{J}_{R, \text { rarm }}=\partial \boldsymbol{f}_{R}(\boldsymbol{q}) / \partial \boldsymbol{q}_{R}$. 


\section{Problem formulation}

In this paper, the primary task of the humanoid manipulator is to move its end-effectors from the given initial location $\boldsymbol{x}_{0}$ (corresponding to the initial configuration of the robot) to the final location $\boldsymbol{x}_{f}$. Additionally, due to the redundancy of the robot (the Jacobian matrix has more columns than rows), the following secondary objectives are taken into account:

- the motion of the robot has to take into account constraints connected with the obstacles existing in the workspace,

- configuration of the manipulator joints should be far away from singular configurations.

Using the kinematic model of the humanoid manipulator given by dependency (2.1), the primary task of the robot can be expressed as

$$
\boldsymbol{f}(\boldsymbol{q}(T))=\left[\begin{array}{l}
\boldsymbol{f}_{L}(\boldsymbol{q}(T)) \\
\boldsymbol{f}_{R}(\boldsymbol{q}(T))
\end{array}\right]=\left[\begin{array}{l}
\boldsymbol{p}_{L, f} \\
\boldsymbol{p}_{R, f}
\end{array}\right]=\boldsymbol{x}_{f},
$$

where $t=T$ is the final moment of motion, $\boldsymbol{x}_{f}$ is the vector containing the final position of the left $\boldsymbol{p}_{L, f}$ and the right $\boldsymbol{p}_{R, f}$ end-effectors of the robot.

Furthermore, at the initial moment of motion, i.e. for $t=0$, the manipulator is (by assumption) in the collision-free, nonsingular configuration for which its end-effectors are in the initial location $\boldsymbol{x}_{0}$

$$
\boldsymbol{f}(\boldsymbol{q}(0))=\left[\begin{array}{l}
\boldsymbol{f}_{L}(\boldsymbol{q}(0)) \\
\boldsymbol{f}_{R}(\boldsymbol{q}(0))
\end{array}\right]=\left[\begin{array}{l}
\boldsymbol{p}_{L, 0} \\
\boldsymbol{p}_{R, 0}
\end{array}\right]=\boldsymbol{x}_{0},
$$

where $\boldsymbol{x}_{0}$ is the vector containing the initial position of the left $\boldsymbol{p}_{L, 0}$ and the right $\boldsymbol{p}_{R, 0}$ end-effectors of the robot.

Finally, the motion of the robot should be planned in such a way as to accomplish secondary objectives during the execution of the primary task. Conditions resulting from the constraints connected with the obstacles existing in the workspace can be written as a set of inequalities

$$
\psi_{i}(\boldsymbol{q}(t)) \geq 0, \quad i=1: L,
$$

where: $\psi_{i}$ is a scalar function which describes collision-free conditions of the robot with the obstacles and $L$ stands for the total number of collision avoidance conditions.

In practice, the configuration of the manipulator joints should be far away from singular configurations. In order to achieve this goal, the Yoshikawa manipulability measure (Yoshikawa [11]) which describes the distance to singular configurations may be maximized. The Yoshikawa manipulability index $\mu(\boldsymbol{q})$ is proportional to the volume of the velocity ellipsoid. The semi-axes of this ellipsoid coincide with eigenvectors of the matrix $\boldsymbol{J} \boldsymbol{J}^{T}$, the lengths of these semi-axes are equal to square roots of the eigenvalues of $\boldsymbol{J} \boldsymbol{J}^{T}$. Since the product of semi-axes lengths is proportional to the volume of the ellipsoid and the product of eigenvalues of the matrix is equal to the determinant of the matrix, the manipulability measure may be calculated as: $\mu(\boldsymbol{q})=\sqrt{\operatorname{det}\left(\boldsymbol{J} \boldsymbol{J}^{T}\right)}$. It seems that in the case of a dual arm humanoid manipulator avoiding singular configurations of the robot arms (Dietrich et al. [6]) is important, so the following manipulability measure is taken into account

$$
\mu(\boldsymbol{q})=\sqrt{\operatorname{det}\left(\boldsymbol{J}_{\text {arms }} \boldsymbol{J}_{\text {arms }}^{T}\right)},
$$

where

$$
\boldsymbol{J}_{\text {arms }}=\left[\begin{array}{cc}
\boldsymbol{J}_{L, \text { larm }} & \boldsymbol{0} \\
\boldsymbol{0} & \boldsymbol{J}_{R, \text { rarm }}
\end{array}\right] \text {. }
$$




\section{Solution}

Dependency (3.1) defines the primary task of the robot. Introducing the end-effectors error $\boldsymbol{e}(\boldsymbol{q}(t))$

$$
e(q)=f(q)-x_{f},
$$

the primary task of the robot can be presented in a more general form as

$$
\lim _{t \rightarrow \infty} \boldsymbol{e}(\boldsymbol{q}(t))=\mathbf{0}
$$

In order to solve the above problem the following differential equation is proposed

$$
\dot{\boldsymbol{e}}+\boldsymbol{K} \boldsymbol{e}=\boldsymbol{0},
$$

where $\boldsymbol{K}$ is a diagonal matrix with positive gain coefficients.

As can be easily shown, the system (4.3) is asymptotically stable (for the diagonal matrix $\boldsymbol{K}$ with positive coefficients) so the end-effectors error $\boldsymbol{e}$ converges to zero. The convergence rate depends on the gain coefficients of matrix $\boldsymbol{K}$ - the larger values of coefficients, the faster the convergence (see e.g. Siciliano et al. [12]). After substituting the derivative of error $\boldsymbol{e}$ into (4.3) the trajectory of the robot can be obtained from

$$
\boldsymbol{J} \dot{\boldsymbol{q}}+\boldsymbol{K} \boldsymbol{e}=\boldsymbol{0} .
$$

The humanoid manipulator has redundant degrees of freedom (its Jacobian matrix has more columns than rows) so generalized velocities of the robot joints cannot be obtained directly from Eq.(4.4). If the error measure $\boldsymbol{e}$ and Jacobian matrix $\boldsymbol{J}$ are given for a certain configuration $\boldsymbol{q}$, joint velocities $\dot{\boldsymbol{q}}$ satisfying Eq.(4.4) can be found by minimizing the following cost function

$$
L(\dot{\boldsymbol{q}}, \lambda)=\frac{1}{2} \dot{\boldsymbol{q}}^{T} \dot{\boldsymbol{q}}+\lambda(\boldsymbol{J} \dot{\boldsymbol{q}}+\boldsymbol{K} \boldsymbol{e}),
$$

where the first term in this function is responsible for minimizing the joints velocities and the second term is responsible for fulfilling constraints (4.4), $\lambda$ is the vector of unknown Lagrange multipliers which make it possible to fulfill constraints (4.4).

The optimal joints velocity vector $\dot{\boldsymbol{q}}$ has to satisfy necessary conditions

$$
\frac{\partial L}{\partial \dot{\boldsymbol{q}}}=\mathbf{0}, \quad \frac{\partial L}{\partial \lambda}=\mathbf{0}
$$

which for the function (4.5) can be written as

$$
\dot{\boldsymbol{q}}+\boldsymbol{J}^{T} \boldsymbol{\lambda}=\mathbf{0}, \quad \boldsymbol{J} \dot{\boldsymbol{q}}+\boldsymbol{K} \boldsymbol{e}=\mathbf{0} .
$$

Solving the first equation for $\dot{\boldsymbol{q}}$ and substituting it into the second one, $\boldsymbol{\lambda}$ is obtained as

$$
\boldsymbol{\lambda}=\left(\boldsymbol{J} \boldsymbol{J}^{T}\right)^{-1} \boldsymbol{K} \boldsymbol{e}
$$

Finally, substituting $\lambda$ into the first equation, the optimal velocities $\dot{\boldsymbol{q}}$ of the robot can be determined as

$$
\dot{\boldsymbol{q}}=-\boldsymbol{J}^{\#} \boldsymbol{K} \boldsymbol{e},
$$

where $\boldsymbol{J}^{\#}$ is the Moore-Penrose pseudoinverse of matrix $\boldsymbol{J}, \boldsymbol{J}^{\#}=\boldsymbol{J}^{T}\left(\boldsymbol{J} \boldsymbol{J}^{T}\right)$. 
In order to fulfill secondary objectives of the task, the following dependency (resulting from properties of $\boldsymbol{J}^{\#}$ ) is used

$$
\boldsymbol{J} \boldsymbol{J}^{\#}=\boldsymbol{I},
$$

where $\boldsymbol{I}$ is the identity matrix.

Right multiplying the above dependence by $\boldsymbol{J}$ gives

$$
\boldsymbol{J}\left(\boldsymbol{I}-\boldsymbol{J} \boldsymbol{J}^{\#}\right)=\boldsymbol{0}
$$

where $\boldsymbol{I}-\boldsymbol{J} \boldsymbol{J}^{\#}$ is the projection matrix on the null space $\boldsymbol{J}$, and as a result: if $\dot{\boldsymbol{q}}^{*}$ is a solution of Eq.(4.4) then for any chosen vector $\dot{\boldsymbol{q}}_{0}$, a solution of Eq.(4.4) is also a vector

$$
\dot{\boldsymbol{q}}=\dot{\boldsymbol{q}}^{*}+\left(\boldsymbol{I}-\boldsymbol{J} \boldsymbol{J}^{\#}\right) \dot{\boldsymbol{q}}_{0}
$$

Finally, it may be concluded that the vector $\dot{\boldsymbol{q}}_{0}$ allows changing the configuration of the robot without changing positions of its end-effectors. It may be used to satisfy the secondary objectives of the task. According to Siciliano et al. [12] it is usually taken as

$$
\dot{\boldsymbol{q}}_{0}=c\left(\frac{\partial w(\boldsymbol{q})}{\partial \boldsymbol{q}}\right)^{T}
$$

where $w(\boldsymbol{q})$ is the secondary objective function and $c$ is a positive coefficient describing a gain connected to this function.

In this paper, two secondary tasks are taken into account, so the following form of vector $\dot{\boldsymbol{q}}_{0}$ is proposed

$$
\dot{\boldsymbol{q}}_{0}=c_{\mu}\left(\frac{\partial \mu(\boldsymbol{q})}{\partial \boldsymbol{q}}\right)^{T}-c_{\kappa} \sum_{i=1}^{L}\left(\frac{\partial \kappa\left(\psi_{i}(\boldsymbol{q})\right)}{\partial \boldsymbol{q}}\right)^{T}
$$

where $c_{\mu}$ and $c_{\kappa}$ are gain coefficients connected to maximization of the manipulability measure of the robot and minimization of the penalties related with collision avoidance constraints, $\kappa$ is a penalty function which associates a penalty with a violation of collision avoidance constraint. In the numerical example it has been taken as

$$
\kappa\left(\psi_{i}(\boldsymbol{q})\right)= \begin{cases}\left(\psi_{i}(\boldsymbol{q})-\varepsilon\right)^{2} & \text { for } \psi_{i}(\boldsymbol{q}) \leq \varepsilon, \\ 0 & \text { otherwise }\end{cases}
$$

where $\varepsilon$ is a constant positive coefficient determining the threshold value which activates the constraint. Finally, the trajectory $\boldsymbol{q}(t)$ that satisfies boundary conditions (3.1) and (3.2), collision avoidance constraints (3.3) and maximizes the performance index (3.4), can be obtained from Eq.(4.6) taking into account (4.7) and (4.8) as follows

$$
\dot{\boldsymbol{q}}=-\boldsymbol{J}^{\#} \boldsymbol{K}\left(\boldsymbol{f}(\boldsymbol{q})-\boldsymbol{x}_{f}\right)+\left(\boldsymbol{I}-\boldsymbol{J} \boldsymbol{J}^{\#}\right)\left(c_{\mu}\left(\frac{\partial \mu(\boldsymbol{q})}{\partial \boldsymbol{q}}\right)^{T}-c_{\kappa} \sum_{i=l}^{L}\left(\frac{\partial \kappa\left(\psi_{i}(\boldsymbol{q})\right)}{\partial \boldsymbol{q}}\right)^{T}\right) .
$$

\section{Numerical example}

In the numerical example, a humanoid manipulator, working in a three-dimensional task space was considered. The kinematic parameters of the robot are given as (all physical values are expressed in the SI system) 


$$
l_{T_{1}}=2.0, \quad l_{T_{2}}=l_{T_{3}}=0.5, \quad l_{L_{1}}=l_{L_{2}}=l_{L_{3}}=l_{R_{1}}=l_{R_{2}}=l_{R_{3}}=1.0 .
$$

At the initial moment of motion the robot is in the nonsingular, the collision-free configuration

$$
\boldsymbol{q}(0)=\left[\begin{array}{llllllllllll}
0, & 0, & \frac{1}{3} \pi, & \frac{1}{4} \pi, & 0, & 0, & 0, & 0, & 0, & 0, & 0, & 0
\end{array}\right]^{T} .
$$

The task of the humanoid manipulator is to move its end-effectors from initial positions

$$
\boldsymbol{p}_{L, 0}=\left[\begin{array}{lll}
0.12, & -2.23, & 3.05
\end{array}\right]^{T}, \quad \boldsymbol{p}_{R, 0}=\left[\begin{array}{lll}
0.12, & 2.23, & 3.05
\end{array}\right]^{T},
$$

to the final positions

$$
\boldsymbol{p}_{L, f}=\left[\begin{array}{lll}
-2.0, & 2.0, & 4.0
\end{array}\right]^{T}, \quad \boldsymbol{p}_{R, f}=\left[\begin{array}{lll}
-2.0, & 4.0, & 4.0
\end{array}\right]^{T} .
$$

There are two obstacles in the workspace, each of them is approximated by the sphere, their center points and radiuses are given as follows

$$
\boldsymbol{p}_{\text {obst }, 1}=\left[\begin{array}{lll}
-1.0, & 2.0, & 3.0
\end{array}\right]^{T}, \quad \boldsymbol{p}_{\text {obst }, 2}=\left[\begin{array}{lll}
-1.0, & 4.0, & 3.0
\end{array}\right]^{T}, \quad r_{\text {obst }, 1}=r_{\text {obst }, 2}=0.25 .
$$

where: $\boldsymbol{p}_{\text {obst }, i}$ is the center of $i$-th obstacle and $r_{o b s t, i}$ is the radius of $i$-th obstacle.

Three cases of performing this task have been considered. In the first one, the humanoid manipulator has performed the primary task only. In the second case, the collision avoidance constraints have been taken into account. In the last case, the primary task and both secondary tasks have been considered. In each case the trajectory of the robot has been obtained by integrating velocities (4.9) over time and using initial condition (5.1). It has been assumed that the algorithm stops when the distance to the desired location $\boldsymbol{x}_{f}$ is less than the given value $\varepsilon$, e.g.: $\varepsilon=0.01$. In all simulations the gain coefficients matrix $\boldsymbol{K}$ has been equal to the identity matrix, so in all simulations the rate of convergence is similar and the final time $T$ of task execution in each task has been obtained as $6.4[s]$.

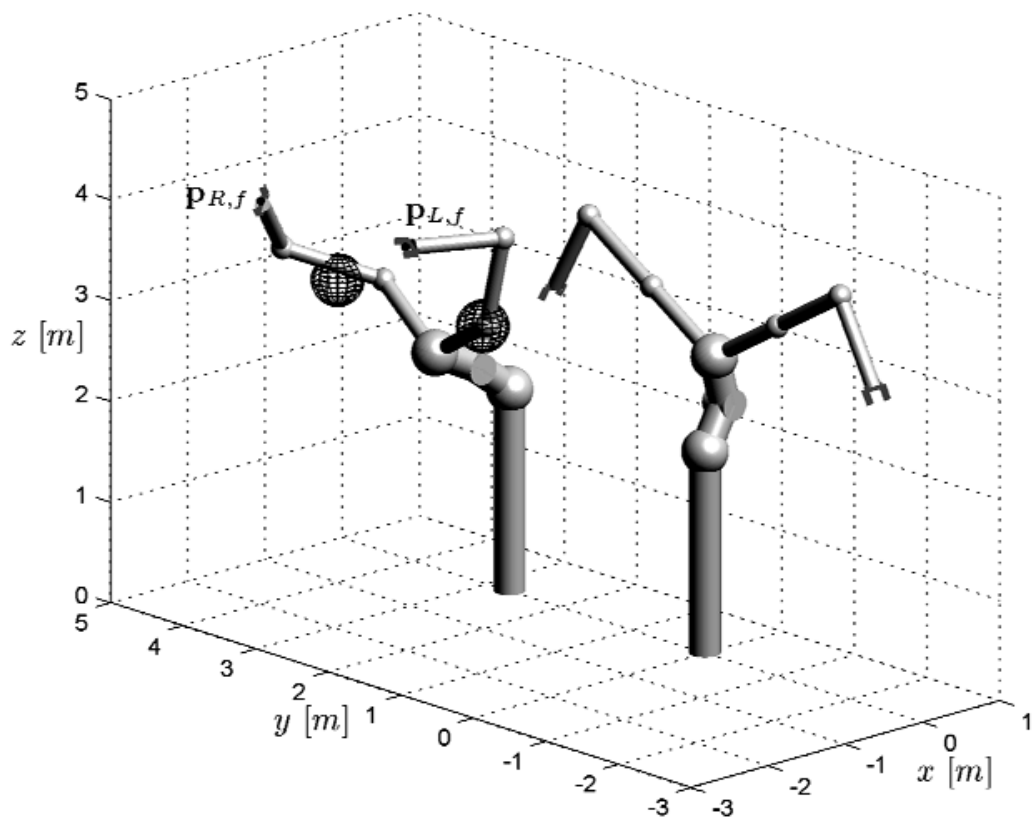

Fig.2. Robot movement without collision avoidance constraints. 
In the first case, the primary task has been considered only, so the gain coefficients connected to the secondary tasks have been taken as: $c_{\mu}=0.0, c_{\mathrm{\kappa}}=0.0$. The initial and final configuration of the manipulator in the workspace, distance to the obstacles and changes of the manipulability measure obtained in numerical simulations, are shown in Figs 2 - 4, respectively. In Fig.2 the obstacles have been shown, and as it can be seen from Fig.2 and Fig.3., the robot may potentially collide with both spheres in the workspace.

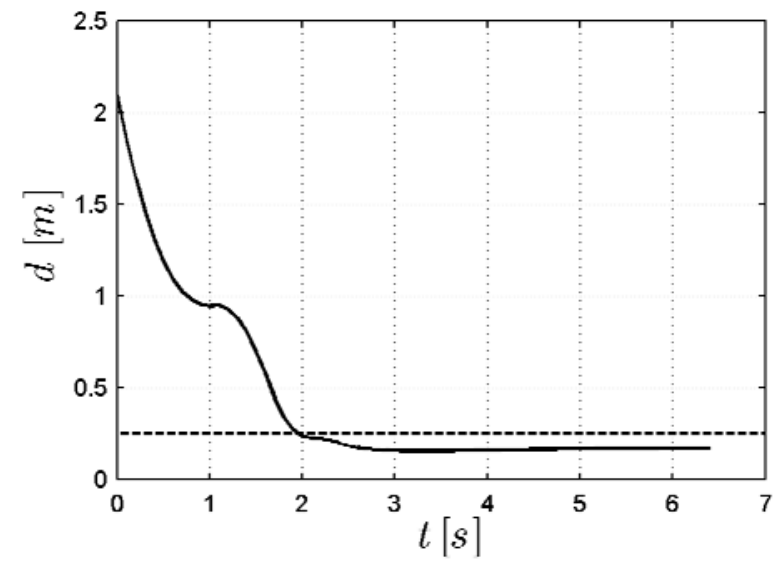

Fig.3. Distance to the obstacles.

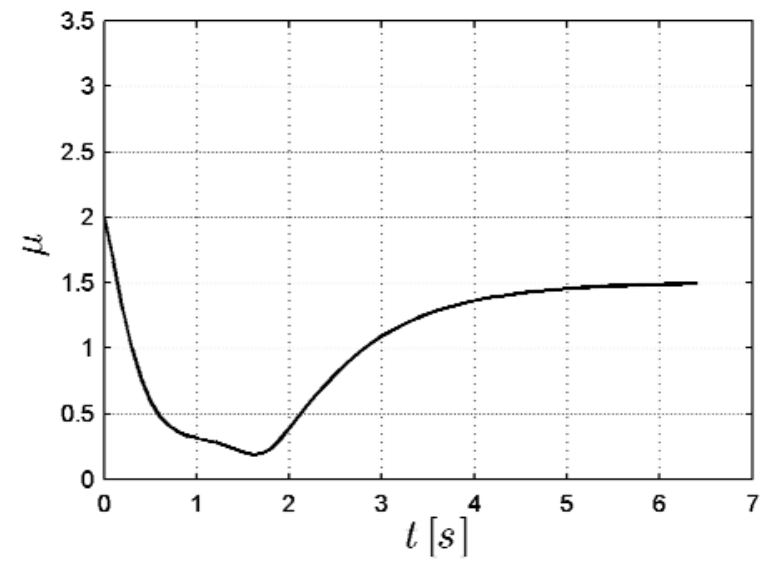

Fig.4. Changes of the manipulability measure.

In the second case collision avoidance conditions have been considered $\left(c_{\mu}=0.0, c_{\kappa}=1.0\right)$. Figures 5 and 6 illustrate how the manipulator avoids collision with the first and the second obstacle, changes of the manipulability measure obtained in this simulation are shown in Fig.7. As it can be seen, robot avoids collisions but the value of the manipulability measure of the robot arms obtained in this simulation is smaller than the one obtained in the first task.

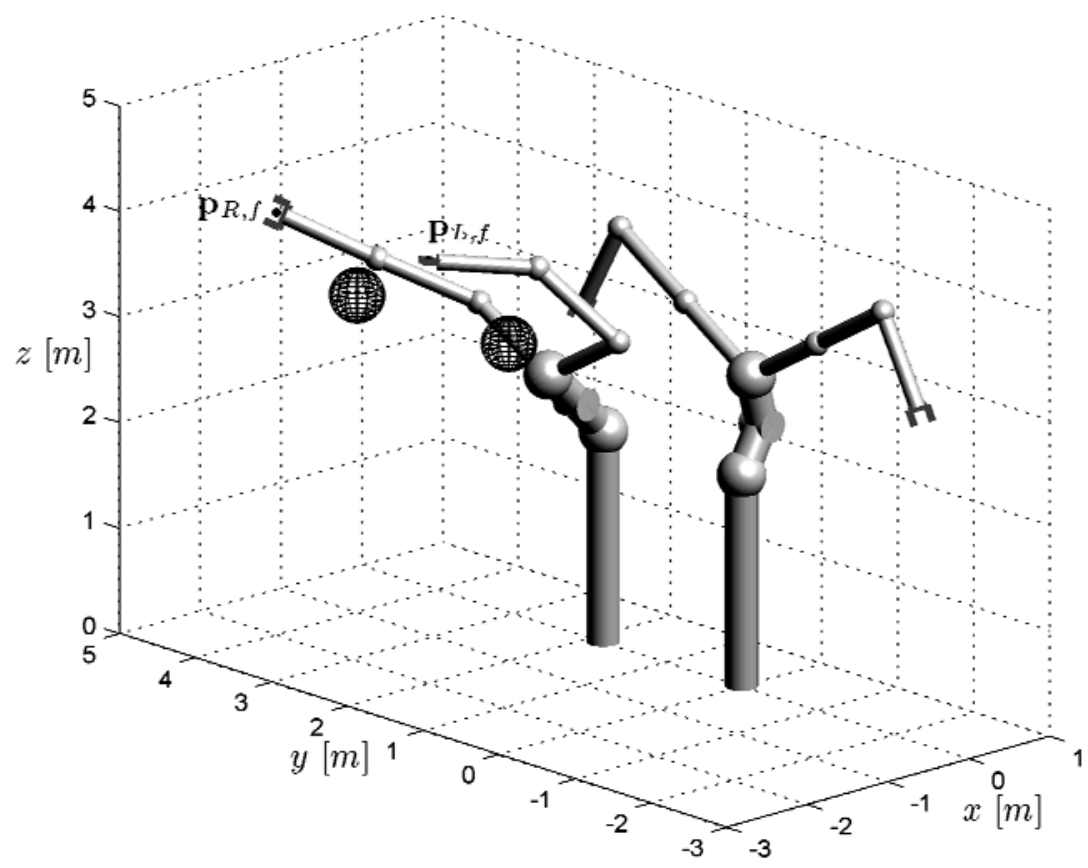

Fig.5. Collision-free robot motion. 


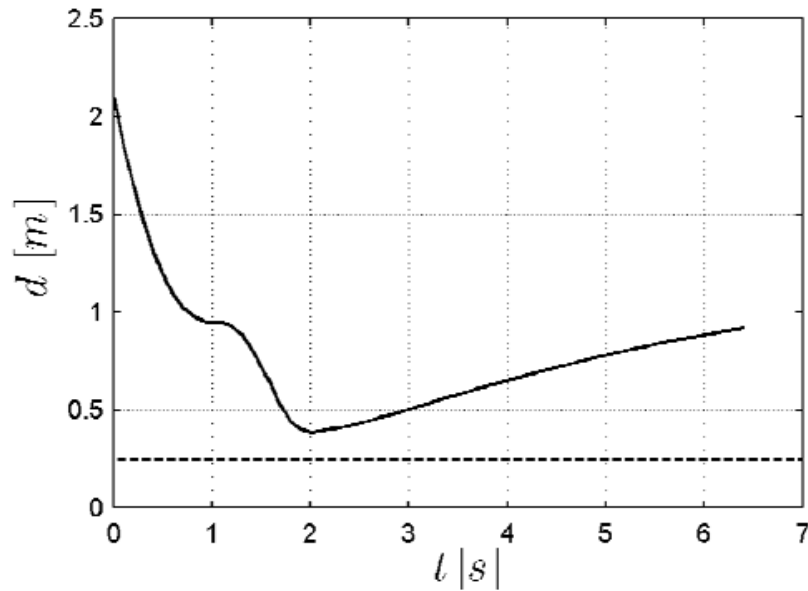

Fig.6. Distance to the obstacles.

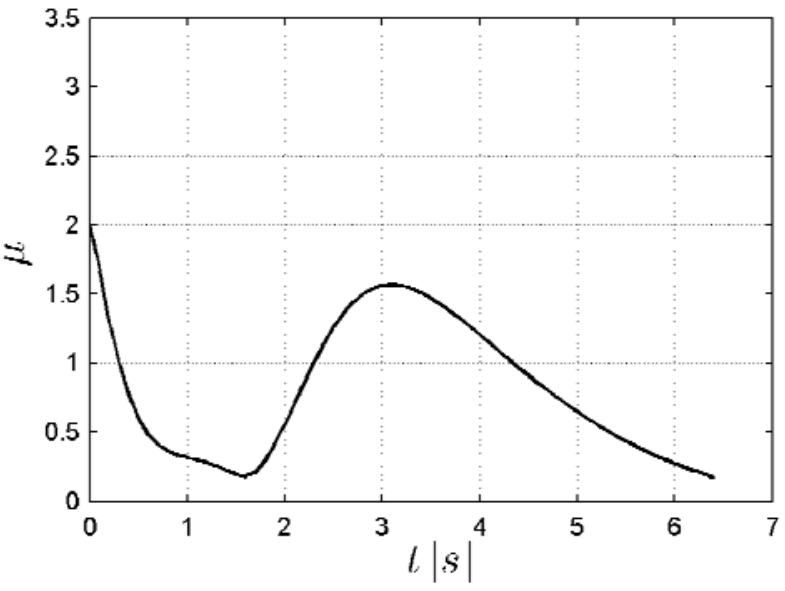

Fig.7. Changes of the manipulability measure.

In the last case both secondary tasks have been considered $\left(c_{\mu}=1.0, c_{\kappa}=1.0\right)$. Figures 8 and 9 present the collision-free manipulator motion. Additionally, as it can be seen from Fig.10, in this case the manipulability measure of the robot achieves the highest value.

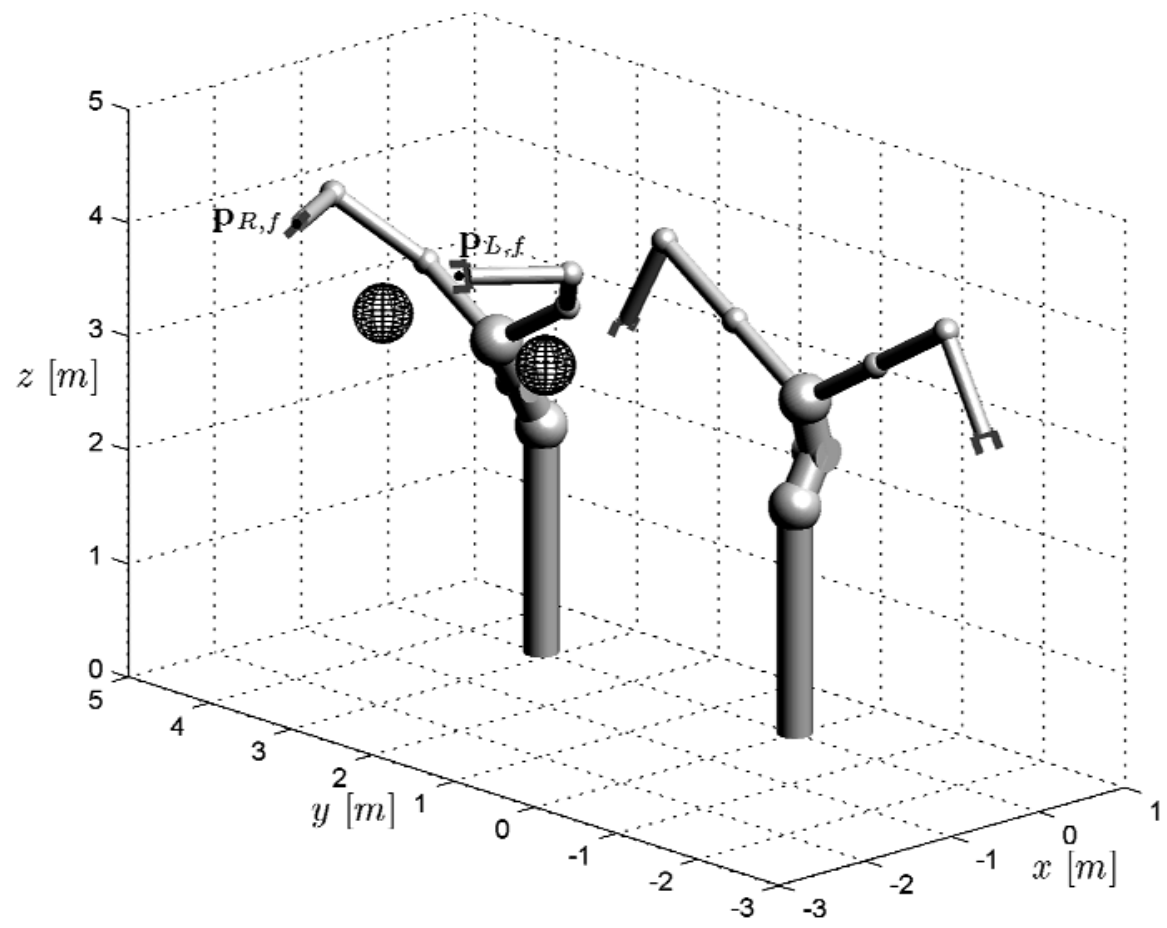

Fig.8. Collision-free robot motion. 


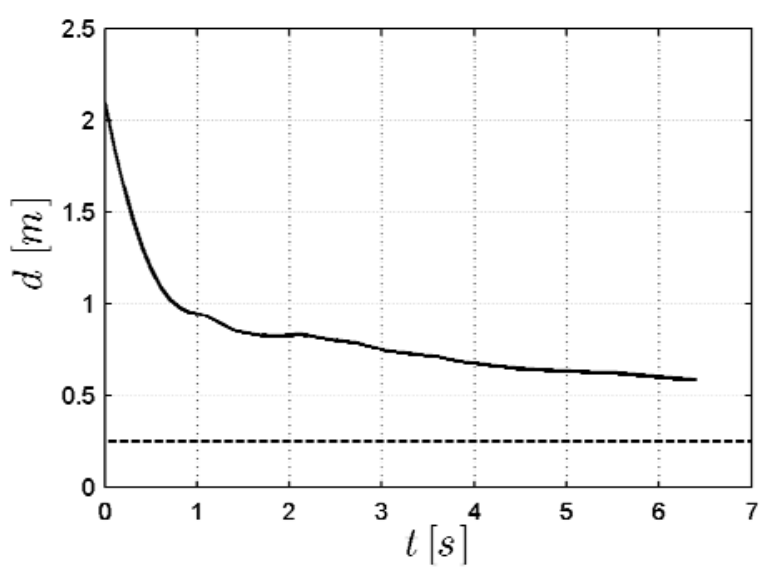

Fig.9. Distance to the obstacles.

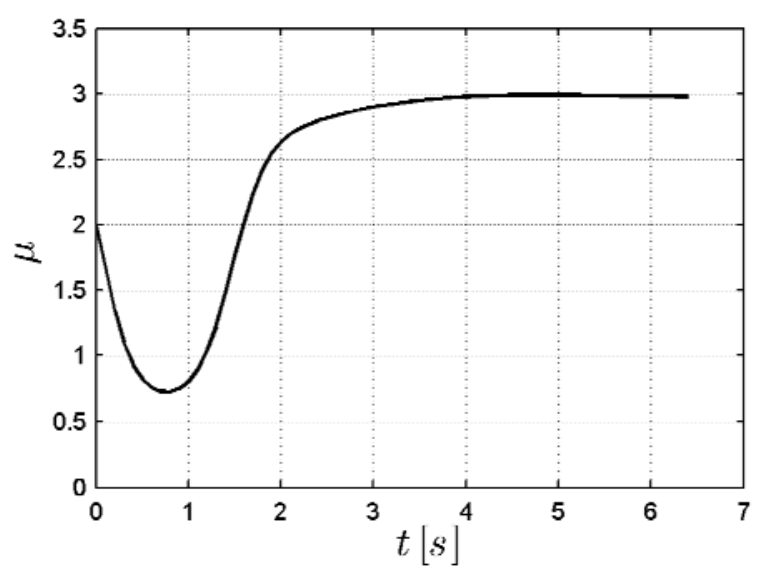

Fig.10. Changes of the manipulability measure.

\section{Conclusions}

In this paper, a method of planning a collision-free trajectory for a humanoid manipulator has been presented. A redundancy resolution approach at the velocity level has been used to solve the task of the manipulator working in the workspace including obstacles. Moreover, the robot motion is planned in the manner to maximize a manipulability measure in order to avoid singular configurations of the robot arms. The proposed approach of the trajectory planning allows real time computations. The effectiveness of the presented method is confirmed by the results of the computer simulations.

A certain disadvantage of the proposed approach is that it allows taking into account boundary conditions dependent on the robot configuration only. For this reason, in subsequent studies the redundancy resolution will be performed at the acceleration level. Our future research will concentrate also on the application of a method to plan the trajectory of the robot solving the dual arm manipulation task. In this case, an additional holonomic constraint resulting from a closed kinematic chain has to be taken into account. It seems that solutions proposed by Pajak [13] for cooperating manipulators may be adopted. Additionally, in future application we will consider mounting a humanoid manipulator on a wheeled mobile platform (Pajak [14]) to increase the workspace of the robotic system. In this case the mobile manipulators can be used as service robots in the productions systems as well as in health care (Pajak and Pajak [15]) or as home assistant (Deitrich et al. [16]).

\section{Nomenclature}

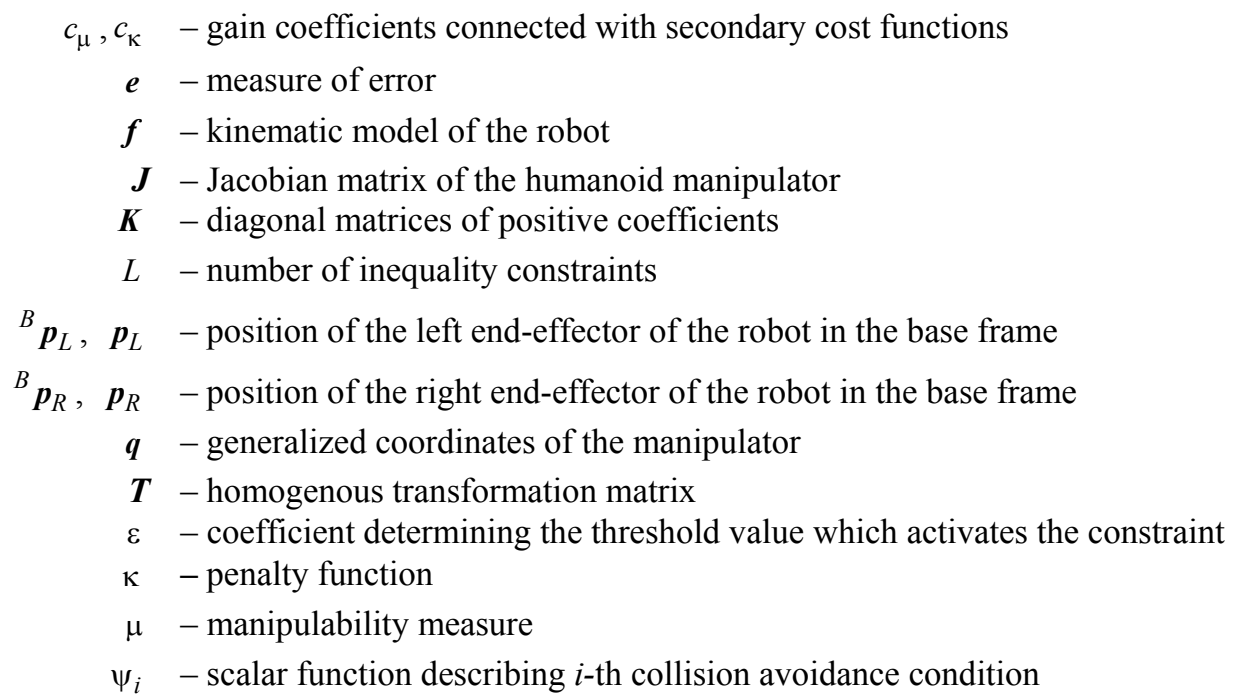




\section{References}

[1] Harada K., Yoshida E. and Yokoi K. (2014): Motion Planning for Humanoid Robots. - Springer Publishing Company, Incorporated.

[2] Caccavale F., Lippiello V., Muscio G., Pierri F., Ruggiero F. and Villani L. (2011): Kinematic control with force feedback for a redundant bimanual manipulation system. - IEEE International Conference on Intelligent Robots and Systems, No.6048549, pp.4194-4200.

[3] Caccavale F., Lippiello V., Muscio G., Pierri F., Ruggiero F. and Villani L. (2013): Grasp planning and parallel control of a redundant dual-arm/hand manipulation system. - Robotica, vol.31, No.7, pp.1169-1194.

[4] Vahrenkamp N., Asfour T., Metta G., Sandini G. and Dillmann R. (2012): Manipulability analysis. - 12th IEEERAS International Conference on Humanoid Robots (Humanoids 2012), Osaka, pp.568-573.

[5] Jamisola R.S., Kormushev P.S., Roberts R.G. and Caldwell D.G. (2016): Task-space modular dynamics for dualarms expressed through a relative Jacobian. - Journal of Intelligent and Robotic Systems: Theory and Applications, vol.83, No.2, pp.205-218.

[6] Dietrich A., Wimböck T. and Albu-Schäffer A. (2011): Dynamic whole-body mobile manipulation with a torque controlled humanoid robot via impedance control laws. - International Conference on Intelligent Robots and Systems, San Francisco, CA, pp.3199-3206.

[7] Caputo A.C., Pelagagge P.M., and Salini P. (2018): Economic comparison of manual and automation-assisted kitting systems. - IFAC-PapersOnLine, vol.51, No.11, pp.1482-1487.

[8] Boudella M.E.A., Sahin E. and Dallery Y. (2017): Modeling kitting operations in a semi-robotic environment. - 7-th IESM Conference.

[9] Patalas-Maliszewska J. and Krebs I. (2016): A model of the tacit knowledge transfer support tool: CKnow-board. - International Conference on Information and Software Technologies, Springer, Cham, pp.30-41.

[10] Craig J.J. (1989): Introduction to Robotics. - Cambridge, Massachusetts: Addison Wesley.

[11] Yoshikawa T. (1985): Manipulability of robotic mechanisms. - International Journal of Robotics Research, vol.4, No.2, pp.3-9.

[12] Siciliano B., Sciavicco L., Villani L. and Oriolo G. (2008): Robotics: Modelling, Planning and Control. Springer Publishing Company, Incorporated.

[13] Pajak I. (2019): Real-time trajectory generation methods for cooperating mobile manipulators subject to state and control constraints. - Journal of Intelligent and Robotic Systems: Theory and Applications, vol.93, No.3, pp.649-668.

[14] Pajak G. (2017): Trajectory planning for mobile manipulators subject to control constraints. - 11th International Workshop on Robot Motion and Control, pp.117-122.

[15] Pajak G. and Pajak I. (2014): Motion planning for mobile surgery assistant. - Acta Bioeng. Biomech., vol.16, No.2, pp.11-20.

[16] Dietrich A., Bussmann K., Petit F., Kotyczka P., Ott C., Lohmann B. and Albu-Schäffer A. (2016): Whole-body impedance control of wheeled mobile manipulators: Stability analysis and experiments on the humanoid robot Rollin' Justin. - Autonomous Robots, vol.40, No.3, pp.505-517.

Received: March 3, 2019

Revised: April 5, 2019 